\title{
Research Progress of Relationship between Intestinal Flora and Allergic Diseases
}

\author{
Weiliang Wang ${ }^{1, *}$ \\ ${ }^{1}$ College of veterinary medicine, Sichuan agricultural university, 611130 Chengdu, China
}

\begin{abstract}
Allergic diseases are the major health problems in the world today. The abnormal structure of intestinal flora is closely related to the occurrence and development of allergic diseases. The formation process of intestinal flora structure in early life is affected by various factors such as delivery mode, feeding mode, addition of probiotics or prebiotics, and heredity, etc. Moreover, the abnormal intestinal flora structure in early life is an important influencing factor for later allergic diseases. In this paper, the relationship between early intestinal flora structure abnormality and the occurrence and development of allergic diseases is discussed in combination with the latest literature.
\end{abstract}

\section{Intestinal microbial composition and influencing factors}

\subsection{Intestinal microbial composition}

In normal adults, the gastrointestinal tract is home to a large number of complex microorganisms, known as intestinal microorganisms. Studies have shown that the adult gastrointestinal tract contains about $500 \sim 1,000$ different kinds of microorganisms, with the number of bacteria being about 1014, 10 times the number of human cells, and the amount of gene encoding is more than 100 times that of human body [1]. Human intestinal microorganisms are mainly divided into 9 Eumycotas, namely Firmicutes, Bacteroidetes, Proteobacteria, Verrucomicrobia, Fusobacteria, Spirochaeates, Cyanobacteria and Vadin BE97. Bacteroidetes and Firmicutes account for more than $90 \%$ of the total intestinal flora. Research generally believes that when the fetus is in the mother's womb, the intestinal tract is nearly sterile. When the fetus is born and exposed to the surrounding environment, bacteria enter the neonatal intestine through the ingestion of vaginal secretion, oral ingestion, skin contact and air inhalation, and rapidly colonize and reproduce in the intestinal tract $[2,3]$. The first colonized microorganisms were mainly staphylococcus, enterobacter, streptococcus and other aerobic bacteria and facultative anaerobic bacteria. With the gradual consumption of oxygen, strict anaerobic bacteria (such as bifidobacteria, bacteroides and clostridium difficile, etc.) gradually replaces the dominant position of aerobic bacteria and facultative anaerobic bacteria and becomes the main dominant flora and reproduces in the intestinal tract [4]. At about 1 year old, its intestinal flora structure is similar to that of adults, and it is gradually improved in the subsequent $1-2$ years to get closer to that of adults [5].

\subsection{Influencing factors of intestinal flora structure}

The structure of intestinal flora is not unchanging, and its formation process is affected by various factors such as delivery mode, feeding mode, growth environment, use of drugs such as antibiotics, addition of probiotics or probiotics, and heredity.

\subsubsection{Delivery mode}

The delivery mode determines the microbial community that the baby is first exposed to after birth, which has a great influence on the colonization of intestinal microorganisms in early infants, especially on the number of bifidobacteria [6-8]. Microorganisms in the mother's birth canal and skin are the main sources of bacterial colonization in the intestinal tracts of infants. Through the analysis of the fecal flora of premature infants, it is found that the microbial structure of the digestive tract of infants is related to the microorganism of the mother's skin (natural delivery) or birth canal (cesarean delivery). A large number of studies have shown that compared with caesarean babies, the colonization time of bacteroides and bifidobacteria in the intestines of natural born infants is earlier [9], the number is higher, and the diversity of intestinal flora is more abundant [8].

\subsubsection{Feeding mode}

Feeding modes has important effects on colonization of intestinal microorganisms in infants. The structure of intestinal flora of infants with different feeding modes is 
obviously different. Studies have shown that artificial feeding can delay the colonization time of bifidobacteria in infants' intestines [10]. In addition, compared with artificially fed infants, infants who are breastfed have more lactobacillus and bifidobacteria in their intestines, and a small amount of clostridium difficile, bacteroides, enterobacter and staphylococcus [11], and the diversity of bifidobacteria in their intestines is also higher [12]. The study has found that the bacterial strains in the feces of breastfed infants are the same as those in the maternal feces, suggesting that the early microorganisms of infants are probably derived from breast milk [13]. Special nutrients in breast milk, such as oligosaccharides, can promote the growth of bifidobacteria and bacteroides, and inhibit the growth of staphylococcus, enterococcus, veillonella, clostridium and enterobacteria. Immune factors and ferritin have strong inhibitory effects on colibacillus and pathogenic bacteria [14]. Bifidobacteria, lactobacillus and streptococcus in breast milk can promote the development of intestinal flora and the maturation of immune system of infants [15].

\subsubsection{Use of antibiotics}

Antibiotics can be used to treat intestinal infectious diseases, but excessive or long-term use can not only kill pathogenic microorganisms, but also kill beneficial bacteria in the intestinal tract, leading to intestinal flora imbalance [16]. The study has indicated that the proportion of enterobacter, enterococcus and klebsiella in infants increases during the period of antibiotic use, but the proportion of bifidobacteria and bacteroides decreases [17]. At the same time, the diversity of intestinal flora also decreases and lasts for a certain period of time [18]. In addition, maternal antibiotic treatment during pregnancy or lactation may reduce the amount of bacteroides and bacteria in the infant's intestinal tract [19].

\subsubsection{Environmental and genetic factors}

The study has analyzed the intestinal microorganisms of children in Germany and Finland, and found that the number of bifidobacteria in the intestinal tracts of Finnish infants is higher than that of German infants [20]. The number of bifidobacteria in the feces of babies born in families with larger numbers of people is higher, but the total number of bacteria is lower. Animal experiments show that the intestinal microflora structure of rats in the same cage presents aggregation, while the intestinal microflora structure of rats in different cages shows significant differences [21].Studies have shown that the intestinal flora colonization and structure of twins are similar, and the number of identical twins is higher than that of fraternal twins [22]. It is shown that the formation of intestinal flora structure of infants is also influenced by environmental factors and genetic factors.

The key period for the formation of intestinal flora structure in infants is before 1 year old, and the formation process of intestinal flora structure is influenced by factors such as delivery mode, feeding mode and the use of antibiotics. Therefore, natural childbirth, breast-feeding and rational and standardized use of antibiotics are conducive to the normal colonization and development of intestinal flora in infants.

\section{Intestinal microorganisms and allergic diseases}

\subsection{Prevalence of allergic diseases}

Allergic diseases are listed as one of the top three diseases in the 21 st century by the world health organization (WHO), and they are also regarded as the major health problems in the world today. Allergic diseases mainly include allergic rhinitis, asthma, atopic dermatitis and allergic eczema. In recent years, with the rapid development of economy and the continuous improvement of people's living standards, allergic diseases have been widely prevalent in the world. A survey in 2003 showed that the incidence of allergic asthma in children under 4 years old in the United States was $160 \%$ higher than that in the 1980s and 1990s [23]. According to the World Allergy Organization (WAO), an epidemiological survey of allergic diseases in 30 countries around the World, about $22 \%$ of the population suffers from different types of allergic diseases. The results of the survey on the prevalence of allergic diseases among children aged $0 \sim 14$ in Beijing, Chongqing and Guangzhou from 2008 to 2009 showed that the prevalence of allergic rhinitis in the three cities was as high as $14.46 \%, 20.42 \%$ and $7.83 \%$. The prevalence of allergic asthma was $3.15 \%, 7.45 \%$ and $2.09 \%$, respectively. The prevalence rate of eczema was $20.64 \%, 10.02 \%$ and $7.22 \%$, respectively [24]. Therefore, it is particularly important to explore the causes of allergic diseases and to prevent them.

\subsection{Intestinal microorganisms and immunity}

Intestinal flora is an important stimulant of the intestinal immune system. By continuously stimulating the local or systemic immune response of the body, intestinal mucosa associated lymphoid tissue (GALT) matures and the barrier function of intestinal mucosa is enhanced. Studies have confirmed that the obligatory anaerobic bacteria, mainly lactobacillus and bifidobacteria, form a biological barrier of intestinal mucosa epithelium by binding with the specific receptor on the intestinal mucosa surface, and assist the organism to resist the invasion of exogenous pathogenic bacteria, which plays a significant role in maintaining the normal immune function of the organism [25]. Although the development of the organism's immune system is basically completed in infancy, its immune function is still not very mature and sound: for example, the phagocytic function of phagocytes is poor. The immune response of the organism at this time is mostly the initial reaction, with slow antibody generation, low titer and weak immune 
ability [26].Therefore, when a baby is born, its intestinal flora is constantly colonized with its contact with the microorganisms in the mother's skin, vagina and the external environment. This process is essential for the differentiation and maturation of the organism's immune system.

\subsection{Intestinal microorganisms and allergic diseases}

\subsubsection{Difference in intestinal flora between allergic children and healthy children}

Studies show differences in intestinal flora between children with allergic constitution and healthy children. Bjrksten et al. [27] analyzed the bacterial flora in the feces of the 2-year old children in 29 cases of Estonia (low incidence region) and 33 cases of Sweden (high incidence region). It was found that the number of lactobacillus and bifidobacteria in the intestines of allergic children was low, while the proportion of aerobic bacteria such as escherichia coli and staphylococcus aureus was increased. And lactobacillus in children's feces in Estonia is more common than that in Sweden. Yong et al. [28] analyzed the type of bifidobacteria in infant feces in high incidence area (UK) and low incidence area (New Zealand)of allergic diseases, and found the infant feces in low incidence area were all found to contain infantile bifidobacteria, while infants in high incidence area were not completely so. Other studies have shown that bifidobacterium longum, bifidobacterium bifidum and pseudomonas bifidum can induce the expression of CD83 in fetal umbilical blood dendritic cells and induce Th2 immune response, and infantile bifidobacteria has no such effect. Kalliomki et al. [29] analyzed the intestinal flora structure of infants using quantitative fluorescence in situ hybridization, and found that clostridium was higher in the feces of allergic infants compared with normal infants, while bifidobacteria was less. In conclusion, in the study of intestinal flora in allergic children and normal children, different intestinal flora structures may be different. However, all the studies on intestinal flora of allergic children suggest that the number of bifidobacteria in feces of allergic children is reduced or the type of bifidobacteria is different from that of normal children.

\subsubsection{The microbial community structure in early life may be an important factor in the development of late allergy}

The etiology of allergic diseases mainly includes genetic factors and environmental factors. As it is impossible for human gene phenotypes to undergo significant variation in a short period of time, the evidence-based data shows that environmental factors are the main reason for the increased incidence of allergic diseases in recent years [30]. "Hygiene hypothesis" [31] believes that the "excessive hygiene" living environment and better hygiene conditions in early life lead to the reduction of microbial exposure, which is closely related to the increased risk of allergic diseases. The natural immunity induced by pathogenic microorganisms can induce the release of Th1 cytokines, while the immune response of newborns is mainly $\mathrm{Th} 2$. So exposure to microorganisms early in life can stimulate the differentiation of Th1 cells of infants, making Th1 / Th2 achieve balance. This can prevent the excessive stimulation of relatively excessive Th2 cytokines to B cells causing increased IgE, thereby reducing the incidence of allergic diseases. On the contrary, the occurrence of allergic diseases may be attributed to the low level of microbial exposure in early life, and the exposure of microorganisms in early life has a decisive effect on the composition of intestinal flora structure [32]. Therefore, the composition of infant intestinal flora structure may be an important factor affecting the development of allergic diseases in later life.

Recent studies have shown that the decrease in the structural diversity of intestinal flora has occurred in children with allergic asthma in early life [32]. Other studies have shown that infants with frequent exposure to endotoxin and more family members have richer intestinal flora structure, while the increased diversity of intestinal flora in early life can prevent the occurrence of allergic diseases or reduce the occurrence of allergic symptoms [33]. The normal colonization of intestinal flora can be affected by delivery mode, feeding mode and the use of antibiotics. Antibiotics can lead to changes in the structure of intestinal flora, resulting in the imbalance of immune homeostasis in the organism, leading to allergic diseases and long-term changes in the structure of intestinal flora [34]. The results of animal experiments have shown that the intestinal flora structure of newborn mice has changed after using vancomycin and are more prone to allergic asthma [35]. Population studies indicate that the occurrence of asthma in later life can be predicted by the species of clostridium XIVa and the subgroup of fragile bacillus in the feces of newborns [36]. A cohort study with large samples in Norway has showed that the incidence of allergic eczema and conjunctivitis at age 3 is lower in children born to mothers who take probiotic dairy products during pregnancy than in the control group [37]. Other studies have shown that probiotics such as lactobacillus and bifidobacteria can improve the composition of intestinal flora structure, and can alleviate allergic symptoms and prevent allergic diseases by inducing the production of Th1 cytokines [38]. Therefore, the intestinal flora structure during infancy and even during pregnancy has a vital influence on the occurrence of late allergic diseases.

\section{Prospects}

In recent years, with the continuous development of intestinal metabonomics, genomics and proteomics, the role of intestinal microorganisms in maintaining the health of the organism has become increasingly clear, and people have gradually realized the importance of maintaining the structural balance of intestinal flora. Infancy is a critical period for the formation of intestinal flora structure, and its formation process is affected by factors such as infant delivery mode, feeding mode and 
the use of antibiotics. A number of studies have shown that the intestinal flora structure of children with allergic diseases is significantly different from that of normal children. It is found that the intestinal flora structure of infants may be closely related to the occurrence of allergic diseases, and the intestinal flora disorder of infants may be an important predictor of allergic diseases in later life. Therefore, we should further explore the relationship between changes of intestinal microorganisms in early life and late allergic diseases, and reduce the incidence risk of allergic diseases through targeted adjustment of intestinal microorganisms. At the same time, probiotics also show positive significance in adjusting the structure of intestinal flora and preventing allergic diseases, providing new research direction for the prevention and treatment of allergic diseases, but large sample studies are still needed to provide evidence support.

\section{References}

1. West CE, Jenmalm MC, Prescott SL. The gut microbiota and its role in the development of allergic disease: a wider perspective $[\mathrm{J}]$. Clin Exp Allergy, 2015, 45(1): 43-53.

2. Zhou D, Zhang H, Bai Z, et al. Exposure to soil, house dust and decaying plants increases gut microbial diversity and decreases serum mmunoglobulin $\mathrm{E}$ levels in BALB / c mice [J]. Environmental Microbiology, 2016, 18(5): 1326-1337.

3. Elgin TG, Kern SL, McElroy SJ. Development of the neonatal intestinal microbiome and its association with necrotizing enterocolitis $[\mathrm{J}]$. Clin Therap, 2016, 38(4): 706-715.

4. Penders J, Gerhold K, Thijs C, et al. New insights into the hygiene hypothesis in allergic diseases: mediation of sibling and birth mode effects by the gut microbiota [J]. Gut Microbes, 2014, 5 (2):239244.

5. Mika M, Mack I, Korten I, et al. Dynamics of the nasal microbiota in infancy: A prospective cohort study [J]. J Allergy Clin Immunol, 2015, 135(4): 905-912.

6. Lee E, Kim BJ, Kang MJ, et al. Dynamics of gut microbiota according to the delivery mode in healthy korean infants [J]. Allergy Asthma Immunol Res, 2016, 8(5): 471-477.

7. Gallego CG, Salminen S. Novel probiotics and prebiotics: how can they help in human gut microbiota dysbiosis? [J]. Appl Food Biotechnol, 2016, 3(2): 72-81.

8. Jakobsson HE, Abrahamsson TR, Jenmalm MC, et al. Decreased gut microbiota diversity, delayed Bacteroidetes colonisation and reduced Th1 responses in infants delivered by caesarean section [J]. Gut, 2014, 63(4): 559-566.

9. Gr nlund MM, Grzekowiak L, Isolauri E, et al. Influence of mother's intestinal microbiota on gut colonization in the infant [J]. Gut Microbes, 2011, 2(4): 227-233.

10. Favier CF, de Vos WM, Akkermans ADL. Development of bacterial and bifidobacterial communities in feces of newborn babies [J]. Anaerobe, 2003, 9(5): 219-229.

11. Fernández L, Langa S, Martín V, et al. The human milk microbiota: origin and potential roles in health and disease [J]. Pharmacol Res, 2013, 69(1): 1-10.

12. Tsuji H, Oozeer R, Matsuda K, et al. Molecular monitoring of the development of intestinal microbiota in Japanese infants [J]. Beneficial Microbes, 2012, 3(2): 113-125.

13. Martín V, Maldonado-Barragán A, Moles L, et al. Sharing of bacterial strains between breast milk and infant feces [J]. J Hum Lact, 2012, 28(1): 36-44.

14. Marcobal A, Barboza M, Froehlich J W, et al. Consumption of human milk oligosaccharides by gut-related microbes [J]. J Agricul Food Chem, 2010, 58(9): 5334-5340.

15. Rescigno M. Intestinal microbiota and its effects on the immune system [J]. Cell Microbiol, 2014, 16(7): 1004-1013.

16. Cox LM, Yamanishi S, Sohn J, et al. altering the intestinal microbiota during a critical developmental window has lasting metabolic consequences $[\mathrm{J}]$. Cell, 2014, 158(4): 705-721.

17. Greenwood C, Morrow AL, Lagomarcino AJ, et al. Early empiric antibiotic use in preterm infants is associated with lower bacterial diversity and higher relative abundance of Enterobacter [J]. J Pediat, 2014, 165(1): 23-29.

18. Schokker D, Zhang J, Vastenhouw SA, et al. Longlasting effects of early-life antibiotic treatment and routine animal handling on gut microbiota omposition and immune system in pigs [J]. PLoS One, 2015, 10(2): e0116523.

19. Korpela K, Salonen A, Virta LJ, et al. Intestinal microbiome is related to lifetime antibiotic use in Finnish pre-school children [J]. Nat Commun, 2016, 7: 10410 .

20. Grzekowiak, Gr nlund MM, Beckmann C, et al. The impact of perinatal probiotic intervention on gut microbiota: double-blind placebo-controlled trials in Finland and Germany [J]. Anaerobe, 2012, 18(1): 713.

21. Lees H, Swann J, Poucher SM, et al. Age and microenvironment outweigh genetic influence on the Zucker rat microbiome [J]. PLoS One, 2014, 9(9): e100916.

22. Goodrich JK, Waters JL, Poole AC, et al. Human genetics shape the gut microbiome [J]. Cell, 2014, 159(4): 789-799.

23. Eichenfield LF, Hanifin JM, Beck LA, et al. Atopic dermatitis and asthma: parallels in the evolution of treatment [J]. Pediatrics, 2003, 111(3): 608-616. 
24. Zhao J, Bai J, Shen KL, et al. Questionnaire-based surveg of allergic diseases among children aged 014 years in the downtown of Beijing, Chongqing and Guangzhou [J]. Chin J Pediatrics, 2011, 49(10): 740744.

25. Hooper LV, Littman DR, Macpherson AJ. Interactions between the microbiota and the immune system [J]. Science, 2012, 336 (6086): 1268-1273.

26. Kau AL, Ahern PP, Griffin NW, et al. Human nutrition, the gut microbiome and the immune system [J]. Nature, 2011, 474 (7351): 327-336.

27. Bj rkstén B, Naaber P, Sepp E, et al. The intestinal microflora in allergic Estonian and Swedish 2-yearold children [J]. Clin Exp Allergy, 1999, 29(3): 342346.

28. Young SL, Simon MA, Baird MA, et al. Bifidobacterial species differentially affect expression of cell surface markers and cytokines of dendritic cells harvested from cord blood [J]. Clin Diagn Lab Immunol, 2004, 11(4): 686-690.

29. Kalliom ki M, Kirjavainen P, Eerola E, et al. Distinct patterns of neonatal gut microflora in infants in whom atopy was and was not developing [J]. J Allergy Clin Immunol, 2001, 107(1): 129-134.

30. Schuijs MJ, Willart MA, Vergote K, et al. Farm dust and endotoxin protect against allergy through A20 induction in lung epithelial cells [J]. Science, 2015, 349(6252): 1106-1110.

31. Jovanovi c' D, Ili c' N, Miljkovi c'-Selimovi B, et al. Campylobacter jejuni infection and $\operatorname{IgE}$ sensitization in up to 2-year-old infants [J]. Vojnosanitetski Pregled, 2015, 72(2): 140-147.

32. Abrahamsson TR, Jakobsson HE, Andersson AF, et al. Low gut microbiota diversity in early infancy precedes asthma at school age [J]. Clin Exp Allergy, 2014, 44(6): 842-850.

33. H rmannsperger G, Clavel T, Haller D. Gut matters: microbe-host interactions in allergic diseases [J]. J Allergy Clin Immunol, 2012, 129(6): 1452-1459.

34. Arrieta M C, Finlay B. The intestinal microbiota and allergic asthma [J]. J Infection, 2014, 69: S53-S55.

35. Russell SL, Gold MJ, Willing BP, et al. Perinatal antibiotic treatment affects murine microbiota, immune responses and allergic asthma [J]. Gut Microbes, 2013, 4(2): 158-164.

36. Vael C, Vanheirstraeten L, Desager KN, et al. Denaturing gradient gel electrophoresis of neonatal intestinal microbiota in relation to the development of asthma [J]. BMC Microbiol, 2011, 11 (1): 1-7.

37. Bertelsen RJ, Brantster AL, Magnus $\mathrm{MC}$, et al. Probiotic milk consumption in pregnancy and infancy and subsequent childhood allergic diseases [J]. Journal of Allergy and Clinical Immunology, 2014, 133(1): 1-8.

38. West CE, Jenmalm MC, Kozyrskyj AL, et al. Probiotics for treatment and primary prevention of allergic diseases and asthma: looking back and moving forward $[\mathrm{J}]$. Exp Rev Clin Imunol, 2016, 12(6): $625-239$. 